\section{Ultrasonografía bronquial con aspiración por aguja fina en el estudio lesiones adyacentes a la vía aérea central}

\author{
SEBASTIÁN FERNÁNDEZ-BUSSY ${ }^{1}$, GONZALO LABARCA ${ }^{\mathrm{a}}$, \\ IVÁN CAVIEDES ${ }^{1}$, ERIK FOLCH ${ }^{2}$, ADNAN MAJID $^{2}$
}

\section{Endobronchial ultrasound and fine needle aspiration for central airway lesions}

\begin{abstract}
Background: Endobronchial ultrasound-guided trans-bronchial needle aspiration (EBUS-TBNA) is a minimally invasive procedure with a high diagnostic yield for lesions adjacent to the central airway. Aim: To describe the diagnostic yield of EBUS-TBNA for lesions suspicious of Non-Small Cell Lung Cancer (NSCLC). Material and Methods: Prospective study of 128 patients aged 25 to 87 years (56\% males) undergoing EBUS TBNA. Radiological features of the lesions were recorded by chest CT scan such as morphology, margins of the lesion, lesion size and location based on the International Association for the Study of Lung Cancer (IASLC) map. Definitive pathological results were evaluated. Results: The average size of lesions was 18.5 millimeter and; 68 cases were of less than 20 millimeters. Sensitivity was $96.7 \%$, specificity $100 \%$, and negative predictive value $93.3 \%$. The most common histological diagnosis was adenocarcinoma. Conclusions: EBUS -TBNA is a useful diagnostic tool for NSCLC suspicious lesions adjacent to the central airway.
\end{abstract}

(Rev Med Chile 2016; 144: 341-346)

Key words: Bronchoscopy; Endosonography; Lung Neoplasms; Ultrasonography, Interventional.

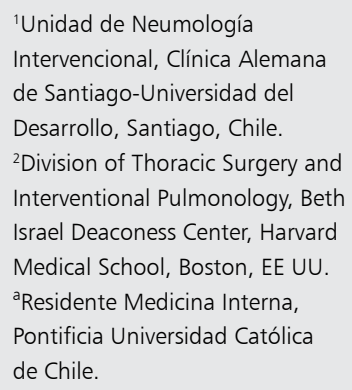

Los autores declaran no tener conflictos de intereses ni recibir algún tipo de financiamiento por el desarrollo del manuscrito.

Recibido el 1 de septiembre de 2015, aceptado el 23 de diciembre de 2015

Correspondencia a:

Dr. Sebastián Fernández-Bussy Avenida Manquehue Norte 1410, Vitacura. Santiago, Chile. Fax: (562) 5754972 sfernandezbussy@alemana.cl
E l enfrentamiento diagnóstico de las lesiones pulmonares sospechosas de cáncer pulmonar ha cambiado en los últimos años, esto debido al desarrollo de técnicas mínimamente invasivas con un alto rendimiento diagnóstico, lo que hace obtener un resultado histológico con mayor rapidez permitiendo iniciar tratamiento de forma más oportuna, en especial en el caso del cáncer pulmonar no células pequeñas ${ }^{1}$.

Clásicamente la cirugía mediante mediastinoscopia, videotoracoscopia y otras técnicas quirúrgicas para diagnóstico y etapificación mediastínica sigue siendo el estándar de referencia. Por otro lado, dentro de las alternativas diagnósticas mínimamente invasivas se encuentra el uso de broncoscopia flexible con biopsia transbronquial, la que tiene un rendimiento cercano a $70 \%$ en lesiones mayores de $30 \mathrm{~mm}$, con una baja tasa de complicaciones. Esta alternativa se encuentra ampliamente disponible en nuestro medio y debiera ser considerada dentro del estudio de lesiones focales mayores de $20 \mathrm{~mm}^{2,3}$. Por otro lado, el estudio de lesiones de menor tamaño mediante alternativas diagnósticas como la ultrasonografía endobronquial (EBUS) con aspiración transbronquial por aguja fina (TBNA), navegación virtual y broncoscopia guiada por navegación electromagnética han permitido obtener muestras histológicas y citológicas representativas con una mayor rapidez y una menor tasa de complicaciones. Estas alternativas han sido evaluadas y son recomendación de estudio de lesiones pulmonares tanto en las guías norteamericanas como europeas ${ }^{1,4,5}$.

Actualmente existen dos tipos de EBUS, el 
lineal y el radial. El EBUS lineal se basa en un broncoscopio con un transductor convexo de ultrasonido en su extremo distal que confirma la ubicación de la lesión en tiempo real, lo que permite generar aspiración del contenido mediante aguja fina (TBNA) aumentando significativamente el rendimiento diagnóstico con una baja tasa de complicaciones asociadas ${ }^{6,7}$.

El objetivo del presente estudio es describir el rendimiento diagnóstico de EBUS lineal como método diagnóstico de lesiones adyacentes a la vía aérea central sospechosas de cáncer pulmonar no células pequeñas.

\section{Material y Método}

Se realizó un estudio prospectivo, de tipo descriptivo donde se incluyeron pacientes con indicación de EBUS-TBNA dentro del estudio diagnóstico de cáncer pulmonar no células pequeñas. Se incluyeron todos los procedimientos realizados de manera consecutiva entre los meses de julio del año 2010 y agosto del año 2013, tanto de pacientes ambulatorios como hospitalizados en Clínica Alemana de Santiago, Chile. Se consideraron como criterios de inclusión aquellos pacientes mayores de 18 años con lesiones adyacentes a la vía aérea central (tanto linfonodos, nódulos o masas pulmonares) con diagnóstico presuntivo de cáncer pulmonar mediante historia clínica y estudio con imágenes (radiografía de tórax, tomografía axial computada (TAC) de tórax y/o tomografía con emisión de positrones (PET) de tórax con lesiones focales sugerentes de cáncer pulmonar). Se excluyeron pacientes con cáncer pulmonar conocido o con imágenes radiológicas sugerentes de cáncer pulmonar con metástasis a distancia (M1 según clasificación TNM) ${ }^{8}$. Además, se excluyeron aquellos que no autorizaron el procedimiento mediante la firma del consentimiento informado.

\section{Protocolo de procedimiento}

Todos los procedimientos fueron realizados por un endoscopista (SF) usando el broncoscopio flexible (videobroncoscopio Olympus ${ }^{\circledR}$, modelo BF -Q180, Miami, Florida. U.S.A) y el broncoscopio con ultrasonido flexible (Olympus ${ }^{\circledR}$, modelo BF-UC180F, Miami, Florida. U.S.A). Los procedimientos se realizaron según las recomendaciones estándares, descritas en la guía del colegio americano de tórax (ACCP) con monitorización y sedación bajo anestesia ${ }^{4}$. Todos los pacientes debieron firmar un consentimiento informado en el que autoriza la realización del procedimiento. Se realizó una inspección completa de la vía aérea mediante un broncoscopio flexible. Posteriormente se realizó EBUS lineal y punción-aspiración de cualquier lesión adyacente a la vía aérea central, obteniendo un mínimo de 6 aspiraciones en cada lesión con sospecha de compromiso neoplásico.

Se registraron variables demográficas tales como edad y género, características de la lesión descritas de acuerdo a TAC de tórax clasificándolas según su ubicación utilizando el mapa mediastínico IASLC ${ }^{9}$, morfología (redonda, oval, triangular, otra), márgenes (definidos o irregular), tamaño de la lesión y complicaciones asociadas al procedimiento. El diagnóstico anatomopatológico de las muestras fue realizado en el servicio de Anatomía Patológica mediante un operador ciego a la historia clínica y resultado diagnóstico del procedimiento previo. Se consideró una muestra positiva para cáncer como diagnóstica, sin tener que realizar una segunda biopsia quirúrgica. En los casos que el EBUS no fuera diagnóstico, se buscó el procedimiento diagnóstico definitivo definido como estándar de referencia (cirugía mediante videotoracoscopia o mediastinoscopia) en un plazo no mayor de 2 meses, mientras que en los casos en los cuales la lesión no fue diagnóstica por ningún método (broncoscópico o quirúrgico), se consideró como estándar de referencia al seguimiento por al menos 12 meses con TAC de tórax.

\section{Análisis de variables}

Los datos se registraron en una base de datos computacional usando el software Excel 2010. Se definieron para este estudio: Verdadero positivo (VP): EBUS-TBNA con muestra positiva para cáncer pulmonar; Verdadero negativo (VN): EBUS-TBNA con muestra negativa para cáncer pulmonar y muestra mediante cirugía negativa para cáncer pulmonar, o con seguimiento de la lesión sin cambios significativos al TAC de Tórax; falso negativo (FN): EBUS-TBNA con muestra negativa para cáncer pulmonar, con cirugía positiva para cáncer pulmonar. En este estudio, no se consideraron procedimientos como falsos positivos, puesto que el resultado histológico es confirmatorio para cáncer de pulmón.

En adición, se definió como rendimiento diag- 
nóstico la sumatoria de aquellos procedimientos mediante EBUS+TBNA con resultado diagnóstico positivo para cáncer y aquellos procedimientos negativos con estándar de referencia negativo para biopsia $(\mathrm{VP}+\mathrm{VN})$.

Con los resultados obtenidos se realizó un estudio de sensibilidad (definida como VP/VP+FN), especificidad (VN/VN+FP) y VPN (VN/VN+FN).

Una vez obtenido el resultado de los procedimientos, se categorizaron los procedimientos positivos como negativos y se analizaron las variables relacionadas con una mayor probabilidad diagnostica, se realizó un análisis de $\mathrm{T}$ de student para variables cuantitativas, y un análisis de chi-cuadrado para variables cualitativas, el resultado fue expresado como odds ratio (OR) con intervalos de confianza (IC) para 95\%. En el caso de las variables cuantitativas se expresó el resultado como diferencias de medias (DM) según el tipo de parámetro analizado, se consideró un valor $\mathrm{p}<0,05$ como estadísticamente significativo. Para esto se utilizó el software SPSS versión 15,0 (SPSS Inc., Chicago. EE UU.)

\section{Resultados}

Durante el período, se incluyeron 128 pacientes consecutivos con indicación de EBUS-TBNA dentro del estudio de lesiones sospechosas de cáncer pulmonar. El 56,2\% de los pacientes eran hombres y la edad promedio fue de 62,6 años (rango 25 a 87 años). El tamaño promedio fue de $18,5 \mathrm{~mm}$ y 68 fueron lesiones menores a $20 \mathrm{~mm}$. El resumen de las características demográficas y de las lesiones de los pacientes incluidos en este estudio se reporta en la Tabla 1.

Respecto a la ubicación, 43,73\% se encontró en región paratraqueal superior o inferior, $16,4 \%$ en región hiliar, $23,43 \%$ subcarínico, $16,4 \%$ en regiones interlobar, lobar y subsegmentario. Dentro de las características ultrasonográficas, 26\% de los presentaron forma oval, 7,8\% triangular y $71 \%$ presentó márgenes regulares. El resumen de la ubicación de las lesiones y del rendimiento diagnóstico se presenta en las Tablas 2 y 3.

Tabla 1. Características demográficas y tamaños de las lesiones

\begin{tabular}{|lcc|}
\hline Total, pacientes & $\mathbf{n}=\mathbf{1 2 8}$ & $\mathbf{( \% )}$ \\
\hline Femeninos & 56 & 43,7 \\
Masculinos & 72 & 56,2 \\
Edad promedio (rango) & 62,6 & $25-87$ \\
Tamaño & & \\
5-9 mm & 4 & 3,1 \\
$10-14 \mathrm{~mm}$ & 36 & 28,1 \\
$15-19 \mathrm{~mm}$ & 28 & 21,8 \\
$20-29 \mathrm{~mm}$ & 57 & 44,5 \\
$>30 \mathrm{~mm}$ & 3 & 2,3 \\
\hline
\end{tabular}

Tabla 2. Localización de lesiones bajo la clasificación IASLC y el resultado de las biopsias realizadas

\begin{tabular}{|ccccc|}
\hline Localización & Muestras & Biopsias positivas & Biopsias negativas & Falsos negativos \\
\hline $2 \mathrm{~L}$ & 3 & 3 & 3 & 0 \\
\hline $2 \mathrm{R}$ & 10 & 8 & 2 & 0 \\
\hline $4 \mathrm{~L}$ & 12 & 9 & 3 & 0 \\
\hline $4 \mathrm{R}$ & 31 & 23 & 8 & 0 \\
\hline 7 & 30 & 19 & 9 & 2 \\
\hline $10 \mathrm{~L}$ & 11 & 9 & 2 & 0 \\
\hline $10 \mathrm{R}$ & 23 & 12 & 10 & 1 \\
\hline $11 \mathrm{R}$ & 3 & 2 & 1 & 0 \\
\hline $11 \mathrm{~L}$ & 2 & 1 & 1 & 0 \\
\hline $12 \mathrm{R}$ & 1 & 1 & 0 & 0 \\
\hline $13 R$ & 2 & 2 & 0 & 0 \\
\hline Total & 128 & 89 & 39 & 3 \\
\hline
\end{tabular}


Tabla 3. Características ultrasonográficas de las lesiones puncionados bajo EBUS-TBNA

\begin{tabular}{|lcr|}
\hline \multicolumn{1}{|c|}{$\mathbf{n = 1 2 8}$} & (\%) \\
Forma & & \\
Oval & 34 & 26,5 \\
Redonda & 61 & 47,6 \\
Triangular & 10 & 7,8 \\
Otra & 23 & 17,9 \\
Márgenes & & \\
Regular & 91 & 71,0 \\
Irregular & 37 & 28,9 \\
\hline
\end{tabular}

El resultado diagnóstico global del estudio mediante EBUS-TBNA mostró una sensibilidad $96,7 \%$, especificidad $100 \%$, valor predictivo negativo $93,3 \%$. De esta manera el rendimiento diagnóstico de EBUS-TBNA fue de 97,6\%.

En 85 de 87 lesiones menores de $20 \mathrm{~mm}$ se logró obtener un resultado, lo que da una sensibilidad de $96,4 \%$ para este grupo y especificidad de $100 \%$.

El diagnóstico definitivo se obtuvo mediante EBUS-TBNA en 98 casos, el uso de técnicas quirúrgicas fue diagnóstico definitivo en 3 casos y el seguimiento radiológico en 36 casos. De los procedimientos mediante EBUS-TBNA que resultaron negativos, 3 de ellos correspondieron a falsos negativos (3 adenocarcinomas pulmonares diagnosticados por técnicas quirúrgicas).

Del total de lesiones pulmonares con diagnóstico mediante algún método, 92 lesiones resultaron ser compatibles con cáncer pulmonar, sin encontrar lesiones benignas. La prevalencia de cáncer pulmonar (de cualquier histología) fue de $71,8 \%$ en nuestra serie, mientras que la prevalencia de cáncer pulmonar no células pequeñas fue de $52,3 \%$. El resumen de los resultados histológicos se reporta en la Tabla 4.

Respecto a las características de las lesiones que resultaron ser positivas, el tamaño de las lesiones con diagnóstico de cáncer pulmonar fue mayor, con una DM de -3,67 (IC -5,91-1,44), p < 0,02, respecto a la edad, no se encontró diferencia significativa, con una DM de -3,96 (IC -9,07-1,14) $\mathrm{p}=0,126$. En el caso de las características ultrasonográficas de las lesiones, el análisis univariado mostró que las lesiones redondas presentaron un mayor riesgo de ser positivas para cáncer pulmo-
Tabla 4. Resultados histológicos definitivos

\begin{tabular}{|lcc|}
\hline Neoplasia & n $=\mathbf{9 2}$ & (\%) \\
\hline Adenocarcinoma & 45 & 48,9 \\
\hline Escamoso & 20 & 21,7 \\
\hline Metástasis extra pulmonar & 13 & 14,1 \\
Células pequeñas & 11 & 11,9 \\
\hline Indiferenciado & 1 & 1,0 \\
linfoma no Hodking & 1 & 1,0 \\
Neuroendocrino & 1 & 1,0 \\
\hline
\end{tabular}

Tabla 5. Análisis univariado de las variables con riesgo de presentar diagnóstico para CPCP mediante EBUS-TBNA

\begin{tabular}{|lcc|}
\hline Variable & OR (IC) & Valor $\mathbf{p}$ \\
\hline Sexo masculino & $1,04(0,50-2,15)$ & 0,531 \\
\hline Margen irregular & $0,87(0,39-1,90)$ & 0,438 \\
\hline Lesión oval & $0,45(0,20-0,99)$ & $0,038^{*}$ \\
Lesión triangular & $0,20(0,05-0,73)$ & $0,013^{*}$ \\
\hline Lesión redonda & $3,92(1,76-8,72)$ & $<0,001^{*}$ \\
\hline
\end{tabular}

Las variables cualitativas se presentan como Odds ratio (OR) y sus respectivos intervalos de confianza (IC).

nar OR 3,92 (IC 1,76-8,72), en el caso de las lesiones triangulares y ovaladas, estas resultaron tener una menor probabilidad de ser lesiones malignas. El resumen de las características ultrasonográficas analizadas se muestra en la Tabla 5.

Finalmente, en nuestra serie encontramos no reportamos complicaciones en relación al procedimiento.

\section{Discusión}

El rendimiento diagnóstico de este procedimiento cuando se indica dentro del estudio de estructuras cercanas a la vía aérea central es alto.

El rendimiento diagnóstico de esta técnica para el estudio de estructuras cercanas a la vía aérea central o mediastino es alto, en un estudio clínico aleatorizado se comparó el rendimiento de este procedimiento como primera alternativa de 
estudio contra las técnicas quirúrgicas habituales, como resultado se obtuvo que la estrategia de estudio inicial mediante EBUS disminuye en dos semanas el tiempo entre el estudio y la toma de decisión clínica comparada con el protocolo de estudio con imágenes y posterior cirugía, por otro lado, se obtuvo una menor incidencia de toracotomías innecesarias, sin encontrar impacto en la mortalidad, en este estudio EBUS-TBNA presento una sensibilidad de $92 \%$ y especificidad de $100 \%{ }^{10}$.

En nuestra serie el rendimiento diagnóstico de EBUS-TBNA fue alta, especialmente porque la totalidad de las lesiones se centraban en la vía aérea central, con lesiones visualizadas al EBUS. Se evaluó el rendimiento según tamaño y se observó que en aquellas lesiones pequeñas $<$ a $20 \mathrm{~mm}$, el rendimiento seguía siendo bueno. Este hallazgo es una contribución en el enfoque del estudio de lesiones centrales. Mediante el uso de ultrasonido, estas pueden ser encontradas y sometidas a aspiración transbronquial con un rendimiento diagnóstico por sobre $90 \%$.

En Latinoamérica se describen dos referencias sobre el rendimiento de esta técnica, una es brasilera, donde Tedde y cols, describen $57 \%$ de procedimientos diagnósticos mediante EBUS, sin embargo, en esta serie el número de pacientes incluidos fue limitado a 50 pacientes con un total de 201 biopsias en 81 linfonodos o masas mediastínicas mediante EBUS-TBNA para diagnóstico y etapificación. En otro estudio, Fernández Bussy y cols evaluaron el rendimiento diagnóstico de EBUS lineal para la etapificación mediastínica en pacientes con diagnóstico de cáncer pulmonar, encontrando una sensibilidad de $91,1 \%$, especificidad de $100 \%$ y valor predictivo negativo de $92,9 \%{ }^{11,12}$.

Dentro del estudio se analizaron las variables tanto clínicas como ultrasonográficas que pueden contribuir a una mayor probabilidad de diagnóstico de cáncer pulmonar, encontrando que aquellas lesiones positivas para cáncer tienen un mayor tamaño y son de características redondas al aplicar la ultrasonografía, este hallazgo es compatible con la literatura. Fujiwara y cols., analizaron las características ultrasonográficas de 487 pacientes con un total de 1.061 linfonodos con sospecha de cáncer pulmonar, encontrando en el análisis multivariado que las lesiones redondas tienen un riesgo de 3,1 (IC 1,79-5,36, p < 0,001), otras variables estadísticamente significativas fueron la presencia de margen regular, lesiones heterogéneas $\mathrm{y}$ con signos de necrosis en su interior ${ }^{13}$.

Respecto a las complicaciones asociadas a EBUS-TBNA, estas son escasas y por lo general menores. En nuestra serie no reportamos complicaciones asociadas al procedimiento. Dentro de las complicaciones reportadas en la literatura se describe el sangrado auto limitado el más frecuente, con tasas reportadas menores a 5\% de los procedimientos mientras que la tasa de neumotórax es de $1 \%$ y sin presentar eventos fatales, similar a lo encontrado en nuestra serie ${ }^{14,15}$.

Las debilidades de este estudio son, en primer lugar, dentro del diseño no se realizó biopsia quirúrgica confirmatoria para los casos de EBUS-TBNA positivos, tal como lo recomiendan las guías de metodología para estudios de rendimiento diagnóstico ${ }^{16}$. Sin embargo, consideramos que aquellos procedimientos diagnósticos que fueron positivos mediante citología para cáncer pulmonar sin biopsia quirúrgica confirmatoria no debe ser una variable confundente. En segundo lugar, este estudio fue realizado en un centro, con el mismo operador, el rendimiento diagnóstico es una variable que es dependiente de la curva de aprendizaje, lo que puede reducir la aplicabilidad en otros centros con menos experiencia en EBUS-TBNA.

Como conclusión, el uso de EBUS-TBNA es una herramienta con un alto rendimiento diagnóstico, con pocas complicaciones asociadas y debe ser considerada una alternativa en el estudio de lesiones adyacentes a la vía aérea central.

\section{Referencias}

1. Rivera MP, Mehta AC, Wahidi MM. Establishing the diagnosis of lung cancer: Diagnosis and management of lung cancer, 3rd ed: American College of Chest Physicians evidence-based clinical practice guidelines. Chest 2013; 143 (5 Suppl): e142S-65S.

2. Fernández-Bussy S, Labarca G, Canals S, Zagollin M, Oyonarte MM, Isamit D, et al. [Bronchoscopy with transbronchial biopsy for the diagnosis of potentially malignant pulmonary lesions: experience in $261 \mathrm{pa}-$ tients]. Rev Med Chile 2015; 143 (4): 433-8.

3. Fernández-Bussy S, Labarca G, Zagolin M, Oyonarte $\mathrm{M}$, Isamit D, Jalilie A, et al. [Immediate complications following flexible bronchoscopy: retrospective analysis of 1079 procedures]. Rev Med Chile 2014; 142 (3): 299304. 
4. Ernst A, Silvestri GA, Johnstone D, American College of Chest P. Interventional pulmonary procedures: Guidelines from the American College of Chest Physicians. Chest 2003; 123 (5): 1693-717.

5. Vilmann P, Clementsen PF, Colella S, Siemsen M, De Leyn P, Dumonceau JM, et al. Combined endobronchial and oesophageal endosonography for the diagnosis and staging of lung cancer. The European respiratory journal 2015; 46 (1): 40-60.

6. Anantham D, Koh MS, Ernst A. Endobronchial ultrasound. Respiratory medicine 2009; 103 (10): 1406-14.

7. Dincer HE. Linear EBUS in staging non-small cell lung cancer and diagnosing benign diseases. Journal of bronchology \& interventional pulmonology 2013; 20 (1): 66-76.

8. Tanoue LT, Detterbeck FC. New TNM classification for non-small-cell lung cancer. Expert review of anticancer therapy 2009; 9 (4): 413-23.

9. Rusch VW, Asamura H, Watanabe H, Giroux DJ, Rami-Porta R, Goldstraw P, et al. The IASLC lung cancer staging project: a proposal for a new international lymph node map in the forthcoming seventh edition of the TNM classification for lung cancer. Journal of thoracic oncology: official publication of the International Association for the Study of Lung Cancer 2009; 4 (5): 568-77.

10. Navani N, Nankivell M, Lawrence DR, Lock S, Makker $\mathrm{H}$, Baldwin DR, et al. Lung cancer diagnosis and staging with endobronchial ultrasound-guided transbronchial needle aspiration compared with conventional approaches: an open-label, pragmatic, randomised controlled trial. The Lancet Respiratory medicine 2015.
11. Tedde ML, Figueiredo VR, Terra RM, Minamoto H, Jatene FB. Endobronchial ultrasound-guided transbronchial needle aspiration in the diagnosis and staging of mediastinal lymphadenopathy: initial experience in Brazil. Jornal brasileiro de pneumologia: publicacao oficial da Sociedade Brasileira de Pneumologia e Tisilogia 2012; 38 (1): 33-40.

12. Fernández-Bussy S, Labarca G, Canals S, Caviedes I, Folch E, Majid A. Diagnostic yield of endobronchial ultrasound-guided transbronchial needle aspiration for mediastinal staging in lung cancer. Jornal brasileiro de pneumologia : publicacao oficial da Sociedade Brasileira de Pneumologia e Tisilogia 2015; 41 (3): 219-24.

13. Fujiwara T, Yasufuku K, Nakajima T, Chiyo M, Yoshida S, Suzuki M, et al. The utility of sonographic features during endobronchial ultrasound-guided transbronchial needle aspiration for lymph node staging in patients with lung cancer: a standard endobronchial ultrasound image classification system. Chest 2010; 138 (3): 641-7.

14. Vilmann P, Puri R. The complete "medical" mediastinoscopy (EUS-FNA + EBUS-TBNA). Minerva medica 2007; 98 (4): 331-8.

15. von Bartheld MB, van Breda A, Annema JT. Complication rate of endosonography (endobronchial and endoscopic ultrasound): a systematic review. Respiration; international review of thoracic diseases. 2014; 87 (4): 343-51.

16. Bossuyt PM, Reitsma JB, Bruns DE, Gatsonis CA, Glasziou PP, Irwig LM, et al. The STARD statement for reporting studies of diagnostic accuracy: explanation and elaboration. Annals of internal medicine 2003; 138 (1): W1-12. 